\title{
Wireless Body Area Networks
}

\author{
Ippagunta Sravya \\ $3^{\text {rd }}$ CSE Sree Vidyanikethan Engineering College
}

\begin{abstract}
In today's environment health care monitoring is very important area of research. Wireless Sensor Networks (WSNs) can also be used in various health care applications. Wireless .Body Area Networks (WBANs) are getting developing interest on account of their appropriateness for extensive variety of medicinal and nontherapeutic applications.In this paper we have reviewed various wireless body area network protocols and techniques to for health care application. Existing work of various authors in this context has been presented in this paper.
\end{abstract}

Keywords: Wireless body area networks, Health care Applications, WSN, patient monitoring

\section{INTRODUCTION}

A body area network (BAN), also referred to as a wireless body area network (WBAN) or a body sensor network (BSN), is a wireless network of wearable computing devices. BAN devices may be embedded inside the body, implants, may be surfacemounted on the body in a fixed position Wearable technology or may be accompanied devices which humans can carry in different positions, in clothes pockets, by hand or in various bags.

The field of computer science is constantly evolving to process larger data sets and maintain higher levels of connectivity. At same time, advances in miniaturization allow for increased mobility and accessibility. Body Area Networks represent the natural union between connectivity and miniaturization. A Body Area Network (BAN) is defined formally as a system of devices in close Proximity to a person's body that cooperate for the benefit of the user.

Wireless Sensor Networks (WSNs) are utilized to screen certain parameters in numerous applications like environment checking, habitant observing, combat zone, farming field ,checking and shrewd homes. These remote sensors are scattered in detecting region to screen field. WBAN is new rising sub-field of WSN. A key use of WBAN is wellbeing checking. Remote sensors are set on the human body or embedded in the body to screen fundamental signs like circulatory strain, body temperature, heart rate, glucose level and so on. With the assistance of WBAN innovation, patients are observed at home for more period. Sensors constantly sense information and forward to medicinal server. One of the significant obstructions in WBAN is to energize the batteries. A productive directing convention is required to conquer this issue of energizing batteries. Numerous vitality proficient directing conventions are proposed in WSN innovation. Nonetheless, WSNs and WBANs have distinctive designs, applications and work in various conditions. It is difficult to port WSN steering conventions to WBAN.Hence, vitality effective directing convention for WBAN is required to screen patients for more period. We propose a high throughput, dependable and stable directing convention for WBAN. Sensors for ECG and Glucose level are set close to the sink. Both these sensors have basic information of patient and required least constriction, high unwavering quality and long life thusly; these sensors dependably transmit their information specifically to sink.

\section{HISTORY AND DEVELOPMENT OF BAN}

BAN technology is still an emerging technology, and as such it has a very short history. BAN technology emerges as the natural by productof existing sensor network technology and biomedical engineering. Professor Guang -Zhong Yang was the first person to formally define the phrase "Body Sensor Network" (BSN) with publication of his book .Body Sensor Networksin 2006. BSN technology represents the lower bound of power and bandwidth from the BAN use case scenarios. However, BAN technology is quite flexible and there are many potential uses for BAN technology in addition to BSNs. The WPAN working group realized the need for a standard for use with devices inside and around close proximity to the human body. IEEE 802.15 established Task Group \#6 to develop the standards for BAN. The BAN task group has drafted a (private) standard that en compasses a large range of possible devices. In this way, the task group has given application and device developers the decision of how to balance data rate and power. 


\section{SYSTEM ARCHITECTURE}

The proposed wireless body area sensor network for health monitoring integrated into a broader multi-tier telemedicine system is illustrated in Figure 1. The telemedical system spans a network comprised of individual health monitoring systems that connect through the Internet to a medical server tier that resides at the top of this hierarchy. The top tier, centered on a medical server, is optimized to service hundreds or thousands of individual users, and encompasses a complex network of interconnected services, medical personnel, and healthcare professionals. Each user wears a number of sensor nodes that are strategically placed on her body. The primary functions of these sensor nodes are to unobtrusively sample vital signs and transfer the relevant data to a personal server through wireless personal network implemented using ZigBee
(802.15.4) or Bluetooth (802.15.1). The medical server keeps electronic medical records of registered users and provides various services to the users, medical personnel, and informal caregivers. It is the responsibility of the medical server to authenticate users, accept health monitoring session uploads, format and insert this session data into corresponding medical records, analyze the data patterns, recognize serious health anomalies in order to contact emergency care givers, and forward new instructions to the users, such as physician prescribed exercises. The patient's physician can access the data from his/her office via the Internet and examine it to ensure the patient is within expected health metrics (heart rate, blood pressure, activity), ensure that the patient is responding to a given treatment or that a patient has been performing the given exercises.

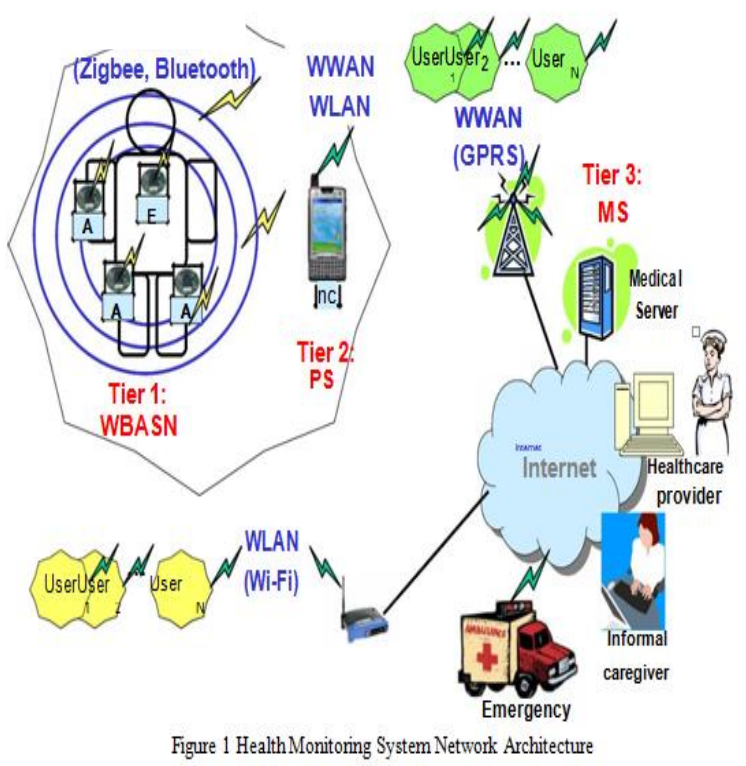

A server agent may inspect the uploaded data and create an alert in the case of a potential medical condition. The large amount of data collected through these services can also be utilized for knowledge discovery through data mining. Integration of the collected data into research databases and quantitative analysis of conditions and patterns could prove invaluable to researchers trying to link symptoms and diagnoses with historical changes in health status, physiological data, or other parameters (e.g., gender, age, weight). In a similar way this infrastructure could significantly contribute to monitoring and studying of drug therapy effects.

The second tier is the personal server that interfaces WBAN sensor nodes, provides the graphical user interface, and communicates with services at the top tier. The personal server is typically implemented on a PDA or a cell phone, but alternatively can run on a home personal computer. This is particularly convenient for in-home monitoring of elderly patients. The personal server interfaces the WBAN nodes through a network coordinator (nc) that implements ZigBee or Bluetooth connectivity. To communicate to the medical server, the personal server employs mobile telephone networks (2G, GPRS, 3G) or WLANs to reach an Internet access point.The interface to the WBAN includes the network configuration and management. The network configuration encompasses the following tasks: sensor node registration (type and number of sensors), initialization (e.g., specify sampling frequency and mode of operation), customization (e.g., run user-specific calibration or user-specific signal processing procedure upload), and setup of a secure communication (key exchange). Once the WBAN network is configured, the personal server manages the network, taking care of channel sharing, time synchronization, data retrieval and processing, 
and fusion of the data. Based on synergy of information from multiple medical sensors the PS application should determine the user's state and his

\section{CASESTUDY}

In this section we present a hypothetical case study to illustrate the usefulness of our proposed system. The patient presented is fictitious, but representative of common issues a recovering heart attack patient would face. We discuss the issues and describe how our system can be used to both address the problem and provide advantages over typical present day solutions. Juan Lopez is recovering from a heart attack. After the release from the hospital he attended supervised physical rehabilitation for several weeks. His physicians prescribed an exercise regime at home. During the physical rehabilitation it was easy to monitor Juan and verify he completed his exercises. Sadly, when left to his own self-discipline, he does not rigorously follow the exercise as prescribed. He exercises, but is not honest to himself (or his physician) as to the intensity and duration of the exercise. As a result, Juan's recovery is slower than expected which raises concerns about his health prognosis, and his physician has no quantitative way to verify Juan's adherence to the program. Our health monitoring system offers a solution for Juan. Equipped with a WBAN, tiny sensors provide constant observation of vital statistics, estimate induced energy expenditure, and assist Juan's or her health status and provide feedback through a user-friendly and intuitive graphical or audio user interface.

exercise. Some of the more common use cases for BAN technology are:

-Body Sensor Networks (BSN)

-Sports and Fitness Monitoring

-Wireless Audio

-Mobile Device Integration

-Personal Video Devices

\section{ISSUES:}

\section{i)Signal \& Path Performance}

As one might expect, the signal and path loss inside the human body is drasticallydifferent than the rules in plain space. That said the rules governing signal and path loss remain the same. Researchers have been able to model signal loss throughout the human body, however the more interesting research involves using the human body as a transmission medium for electrical signals. Marc Wegmueller et al. have attempted to model the conductivity and permittivity of signals sent from one area of the body to another. A full summary of their research is beyond the scope of this paper, but it is worth noting that in the frequency range of $10 \mathrm{kHz}$ to $1 \mathrm{MHz}$, for every $5 \mathrm{~cm}$ between the transmitter and receiver there is an increase in attenuation by 6 to 9 $\mathrm{dB}$. Other factors lowered or raised these constants, such as the geometry of the path, the amount of fat, and the presence of joints.

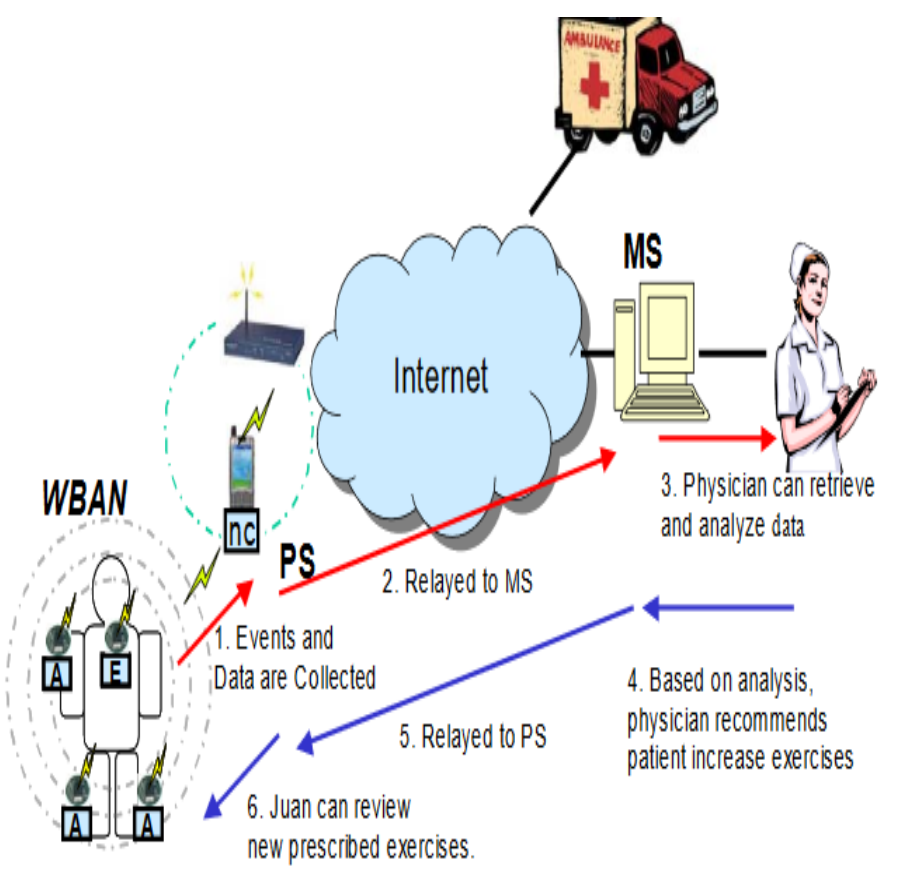

Figure 2 Data flow in the proposed healthcare monitoring system 


\section{APPLICATIONS}

Initial applications of BANs are expected to appear primarily in the healthcare domain, especially for continuous monitoring and logging vital parameters of patients suffering from_chronic diseases such as diabetes, asthma and heart attacks.

- A BAN network in place on a patient can alert the hospital, even before they have a heart attack, through measuring changes in their vital signs.

- A BAN network on a diabetic patient could auto inject insulin through a pump, as soon as their insulin level declines.

Other applications of this technology include sports, military, or security. Extending the technology to new areas could also assist communication by seamless exchanges of information between individuals, or between individual and machines.

\section{i) Managed Body Sensor Networks}

A managed body sensor network (MBSN) is defined as a system where the third party makes decisions based the data collected from one or many BSN. MobiHealth is simply one example of a managed BSN. Harvard University's Code Blue represents another example of BSN currently in the trial stages. Like Mobi Health, Code Blue provides an infrastructure for multiple patient monitoring through EBAN communication.

\section{ii) Autonomous Body Sensor Networks}

Autonomous body sensor networks (ABSN) and MBSN share the same goals, but they accomplish them in different ways. While a MBSN will relies on reading sensor information and delivering it to a third party for decision making and intervention, ABSN take a more proactive approach. ABSN introduce actuators in addition to the sensors to allow the BSN to effect change on the users body. In addition to the actuators, ABSN contain more intelligent sensors that contain enough intelligence to complete their own tasks independently.

\section{iii ) Medical Applications}

WBANs have an immense potential to alter the eventual fate of human service observing by diagnosing numerous life undermining sicknesses and giving ongoing patient checking .In light of advances in innovation (in smaller scale electronic scaling down and combination, sensors, the Internet and remote systems administration) the organization and adjusting of human services administrations will be on a very basic level changed and modernized. The utilization of WBANs is required to expand human services frameworks to empower more successful administration and recognition of diseases, and response to emergency as opposed

\section{CHALLENGES}

BAN technology is still emerging and there are a lot of problems left to solve. Setting aside ethical issues like privacy, there are still plenty of technical challenges that we must overcome before BAN will become an effective solution. The BAN draft submissions have defined solutions for a lot of the basic wireless network protocols, but there is still a large amount of research that must be done to effectively propagate a signal in and around the human body. The last challenge BAN technology faces is actually a problem of Human-Computer Interaction (HCI) and how to make the technology usable.

- Security: Considerable effort would be required to make WBAN transmission secure and accurate. It would have to be made sure that the patient "secure" data is only derived from each patient's dedicated WBAN system and is not mixed up with other patient's data

- Interoperability: WBAN systems would have to ensure seamless data transfer across standards such as Bluetooth, ZigBee etc. to promote information exchange, plug and play device interaction. Further, the systems would have to be scalable, ensure efficient migration across networks and offer uninterrupted connectivity.

- System devices: The sensors used in WBAN would have to be low on complexity, small in form factor, light in weight, power efficient, easy to use and reconfigurable.

- Invasion of privacy: People might consider the WBAN technology as a potential threat to freedom, if the applications go beyond "secure" medical usage.

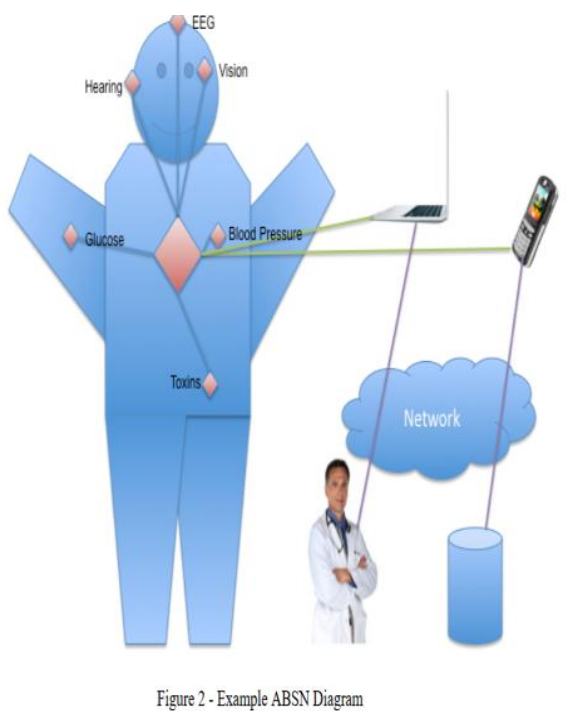


- Sensor validation: Pervasive sensing devices are subject to inherent communication and hardware constraints including unreliable wired/wireless network links, interference and limited .power reserves.

- Data consistency: Data residing on multiple mobile devices and wireless patient notes need to be collected and analysed in a seamless fashion.

- Interference: The wireless link used for body sensors should reduce the interference and increase the coexistence of sensor node devices with other network devices available in the environment.

\section{CONCLUSION}

This paper demonstrates the use of WSNs as a key infrastructure enabling unobtrusive, continual, ambulatory health monitoring. This new technology has potential to offer a wide range of benefits to patients, medical personnel, and society through continuous monitoring in the ambulatory setting, early detection of abnormal conditions, supervised rehabilitation, and potential knowledge discovery through data mining of all gathered information. WBAN systems that monitor vital signs promise ubiquitous, yet affordable health monitoring. We believe that WBAN systems will allow a dramatic shift in the way people think about and manage their health - in the same fashion the Internet has changed the way people communicate to each other and search for information.

\section{REFERENCES}

[1]. https://en.wikipedia.org/wiki/Body_area_n etwork

[2]. http://www.cse.wustl.edu

[3]. http://www. eetimes.com

[4]. Wireless Body Area Networks by Mehmet.R.Yuce.

[5]. Electromagnetics of Body Area Networks: Antennas, Propagation, and RF Systems. 